\title{
India en los márgenes de la gobernanza global: las ambigüedades en la práctica del multilateralismo ${ }^{1}$
}

\section{India at the Margins of Global Governance: A Peculiar Practice of Multilateralism}

\author{
Mario González Castañeda²
}

Resumen: Después de la Guerra Fría, India sometió su política de No alineación a un proceso de autocrítica y de redefinición. Aparentemente, se articuló una nueva Política Exterior: asertiva, pero concomitante con la gobernanza global. No obstante, al examinar el discurso oficial y contrastarlo con la evidencia empírica es posible notar no solo las tensiones y las inestabilidades de la narrativa, sino también la continuidad normativa de la No alineación. El objetivo de este artículo es analizar la práctica del multilateralismo en la Política Exterior india a partir de las experiencias de la SAARC y los BRICS y en perspectiva histórica. El argumento central es que la Política Exterior india caracteriza al multilateralismo y la gobernanza global como limitante del interés y la soberanía nacionales.

Palabras clave: India; Política Exterior; Multilateralismo; Gobernanza Global; SAARC; BRICS.

\begin{abstract}
After the Cold War, India was forced to examine its non-alignment policy. Apparently, a new foreign policy was shaped. It was more ambitious in defining the scope of India's global interests yet, complying with global governance. But despite this appearance, when official discourse is analyzed and contrasted with evidence, it is possible to note not only the tensions and instabilities in the narrative, but also the continuities of non-alignment. This article aims to investigate the practice of multilateralism in Indian foreign policy considering the cases of SAARC and BRICS in historical perspective. The article traces the consistencies in how foreign policy has been conceived: restricting multilateralism as well as global governance, and securing India's national interest and sovereignty.
\end{abstract}

Keywords: India; Foreign Policy; Multilateralism; Global Governance; SAARC; BRICS.

\footnotetext{
1 Recibido: 23/03/17. Aceptado: 14/08/17.

2 Internacionalista por la Universidad del Valle de México; maestro y doctor en Estudios de Asia y África, área: Sur de Asia, por el Centro de Estudios de Asia y África (CEAA), El Colegio de México. Actualmente, realiza una estancia de investigación posdoctoral en el Departamento de Ciencia Sociales de la Universidad Autónoma Metropolitana-Cuajimalpa. mario. gonzalez. castaneda@gmail.com
} 
En 2012, dos think tanks gubernamentales indios: el Indian Defence College y el Centre for Policy Research, patrocinaron la realización de un análisis estratégico de las relaciones internacionales de India intitulado, "No alineación 2. 0: una Política Exterior y estratégica para India en el siglo veintiuno" (Nonalignment 2. 0 A foreign and strategic policy for India in the Twenty First century). El documento fue elaborado por especialistas, académicos y un general en retiro. El objetivo fue sugerir a la Oficina del Primer Ministro el establecimiento de "principios básicos que deben guiar la Política Exterior de India en las décadas por venir" (Khilnani, 2012: iii).

El documento, igualmente, evalúala participación del Estado indio en foros de diálogo y coordinación política y económica, organismos regionales e internacionales. El análisis destaca a los primeros como plataformas coadyuvantes para la ampliación de arenas en las cuales se pueda incidir con fines diversos. Los BRICS (Brasil, Rusia, India, China, Sudáfrica) ${ }^{3}$, foro de coordinación y diálogo financiero, así como la SAARC (South Asia Association for Regional Cooperation), organismo regional-entendidos como herramientas del multilateralismo-no son percibidos en dicho análisis, por tanto, como instrumentos para incrementar el poder de India en la jerarquía internacional, del prestigio ni coadyuvantes para la gobernanza global. Esta evaluación contradice otros estudios en los que se afirma que el Estado indio ha ido incrementando su poder en los procesos de toma de decisiones globales en razón de su participación en dichas iniciativas (Ganguly, 2010; Raja, 2004; Harshe y Seethi, 2005; Pant, 2009). Finalmente, quienes participaron del análisis proponen revitalizar la política de "No alineación".

El estudio refleja una visión cuasi-académica y, en cierto grado semi-oficial, que contradice el discurso histórico con respecto a que los organismos e iniciativas multilaterales: son mecanismos coadyuvantes para el incremento del poder del Estado indio en el Sistema Internacional. Precisamente, al contrastar ese discurso oficial con la práctica del multilateralismo, es posible notar las inestabilidades, las tensiones, las contradicciones y las ambigüedades generadas por el tema. Ese documento representa, así, un excelente punto de partida para explorar la Política Exterior india y la caracterización de la práctica del multilateralismo en ella.

Este artículo tiene por objeto analizar históricamente la práctica del multilateralismo realizada por el Estado indio a partir de las experiencias de un organismo regional y un foro de coordinación: SAARC y los BRICS, respectivamente. El argumento central es que la Política Exterior india ha practicado un multilateralismo sumamente peculiar y reticente a las reconfiguraciones de la gobernanza global. Ello indica la permanencia normativa de la "No alineación". Por lo tanto, se concluirá que la Política Exterior del Estado indio ha caracterizado al multilateralismo y a la gobernanza global en tanto limitante de su interés y soberanía nacionales.

El artículo está dividido en seis apartados. En la introducción se exploran los contextos históricos en los cuales han surgido mecanismos y foros de diálogo y cooperación multilaterales. En el segundo, se analiza conceptualmente el multilateralismo desde distintos enfoques teóricos y cómo estos permiten la gobernanza global. En el tercer

3 El acrónimo BRIC fue ideado originalmente por el corporativo financiero, Goldman Sachs, para agrupar a aquellas economías emergentes con las mejores perspectivas económicas para el siglo XXI: Brasil, Rusia, India y China. Posteriormente, los países así denominados acordaron reunirse para crear un foro de diálogo entre ellos. Sudáfrica fue invitada a incorporarse, resultando en BRICS. 
apartado se examina la articulación histórica de la No alineación en tanto plataforma ideológica y de acción en la Política Exterior india. Posteriormente, se notarán las limitantes teóricas y sus complejidades en la praxis. Para este último fin, en el cuarto apartado se abordan los casos de la SAARC y los BRICS, estableciendo sus objetivos fundacionales y si estos son compatibles con la "No alineación". La participación india en dichos organismos multilaterales es yuxtapuesta y analizada en el quinto apartado para evidenciar las condiciones en las cuales se practica el multilateralismo y si estas coadyuvan a la gobernanza global. Finalmente, se ofrecen conclusiones de esta investigación.

\section{Introducción}

La imperiosa necesidad de encontrar soluciones a problemáticas globales cada vez más complejas ha llevado a la reactivación de las instituciones internacionales "tradicionales", a la creación de foros de diálogo, coordinación y cooperación y, en general, al reforzamiento del multilateralismo. En el contexto de negociaciones internacionales se ha atestiguado el surgimiento de bloques, alianzas políticas y/o estrategias diplomáticas conjuntas Sur-Sur, trascendiendo su espacio fundacional. Ese es el caso de IBSA: India, Brasil y Sudáfrica; estrategia diplomática conjunta surgida originalmente en la ronda preparatoria de las negociaciones de la Ronda de Doha, cuyo objetivo específico ha sido la reducción de los subsidios agrícolas otorgados, principalmente, por Estados Unidos, Japón y la Unión Europea a sus productores, además de la apertura de esos mercados a las exportaciones provenientes de América Latina, Asia y África.

Otro caso similar tuvo lugar en la XV Conferencia de Naciones Unidas sobre Cambio Climático de Copenhague, (Dinamarca), en 2009 (COP15). Brasil, Sudáfrica, India y China (BASIC), presentaron una posición conjunta y distinta a la del resto de los países en desarrollo miembros del Grupo de los Setenta y siete $(G-77)^{4}$, del cual son igualmente parte. El acrónimo de BRICS gradualmente ha sido incorporado al vocabulario de la política y la economía internacionales. Frecuentemente, las agencias de noticias, los periódicos, los noticiarios, los editoriales, los dossiers especiales y las columnas de opinión han empleado dichos acrónimos de forma indiscriminada para referirse a un conjunto de temas y posiciones tan variados en los cuales IBSA, los BASIC o los BRICS pudieron o no haber coincidido.

El denominado fenómeno BRICS o los países BRICS ha comenzado a ser pensado en tanto categoría analítica en las Relaciones Internacionales, a fin de determinar su carácter de alianza, asociación interregional o foro de coordinación y diálogo económico. Algunos autores lo consideran como un nuevo multilateralismo surgido desde el Sur ${ }^{5}$ (Hampson y Heinbecker, 2011;Taylor, 2009; Alden y Vieira, 2005) o una nueva plataforma para la reconfiguración del actual Sistema Internacional, el cual consideran no corres-

\footnotetext{
4 Creado en 1964 por 77 países en desarrollo y miembros de Naciones Unidas, en el marco de la Conferencia de las Naciones Unidas para el Comercio y el Desarrollo (UNCTAD).

5 Se es consciente de las implicaciones, de los alcances y los límites de los conceptos Norte y Sur; no es propósito de este trabajo discutirlos; sin embargo, el primero será empleado para denominar a aquellos Estados con mayor jerarquía de prestigio en el sistema internacional, mientras que el segundo para aquellos con menor jerarquía.
} 
ponde a la realidad política y económica del presente período histórico (Makarychev y Morozov, 2011; Harris, 2005; Pouliot, 2011).

Iniciativas de esa envergadura fueron promovidas en su tiempo, y sus resultados pueden ser juzgados desde distintas perspectivas: el Movimiento de los Países No Alineados (MPNA) y el G-77, por citar dos de los más importantes, resultaron poco eficaces en su cometido, particularmente por la subordinación de su agenda a la dinámica del conflicto ideológico entre Estados Unidos y la Unión Soviética.

La conclusión del conflicto bipolar supuso un período para el fortalecimiento de las instituciones internacionales y, por ende, del multilateralismo. En ese sentido, el conflicto entre Estados Unidos y la Unión Soviética ideologizó, polarizó y llevó a la paralización de la mayor parte de los organismos de Naciones Unidas (particularmente de la Asamblea General y el Consejo de Seguridad) y de sus instituciones subsidiarias (por ejemplo, UNESCO, UNICEF, FAO, UNCTAD ${ }^{6}$ ), tornándolos en espacios de rivalidad. La implosión de la Unión Soviética representó la desideologización y distensión de las relaciones internacionales, propiciando a su vez la reactivación del sistema de Naciones Unidas en tanto mecanismos para la resolución de los problemas de la agenda global.

No obstante, el multilateralismo atravesó por una nueva crisis tras los ataques terroristas en contra de Estados Unidos del 11 de septiembre de 2001. Dichos eventos Ilevaron al gobierno de George W. Bush a rediseñar su estrategia internacional: se percibió un significativo incremento en las amenazas del exterior y, en dicho escenario, Estados Unidos no podía supeditar su seguridad al cumplimiento de acuerdos globales. Por ello, asumió una postura unilateral en sus relaciones internacionales, obligando al resto de la sociedad internacional a fortalecer la cooperación y el multilateralismo.

\section{Multilateralismo y gobernanza global}

El multilateralismo puede ser definido como el diálogo político entre varios Estados con el fin de generar consensos en torno a una temática particular de la agenda global. Ruggie caracteriza al multilateralismo como una forma institucionalizada de coordinación de las relaciones entre tres o más estados sobre la base de principios de conducta 'generalizados' (Ruggie, 1992: 571), y mutuamente definidos. Pouliot señala que este mecanismo de negociación se distingue de las coaliciones o los conciertos ad hoc por ser una práctica amplia, rutinaria y no discriminatoria (Pouliot, 2011).

No obstante, existe una tendencia dogmática en las Relaciones Internacionales, particularmente del Neorrealismo, de considerar al multilateralismo como una forma ineficaz de movilización de los recursos del sistema (Waltz, 1979). En ese sentido, el enfoque teórico Neorrealista argumenta que en un Sistema Internacional descentralizado y anárquico los Estados procuraran la consecución de sus intereses-supervivencia y seguridad-a partir del principio de auto-ayuda, como sugiere Waltz: "El sistema político internacional, como los mercados, está formado y se sustenta en el principio de autoayuda" (Waltz, 1979: 164). Así, la cooperación internacional tiene lugar sólo si las ganan-

6 Organización de las Naciones Unidas para la Educación, la Ciencia y la Cultura; Fondo de las Naciones Unidas para la Infancia; Organización de las Naciones Unidas para la Alimentación y la Agricultura; Conferencia de las Naciones Unidas sobre Comercio y Desarrollo, respectivamente. 
cias son relativas y no absolutas, ya que pretender obtener ganancias absolutas puede hacer al Estado dependiente de los acuerdos involucrados (Waltz, 2000). Asimismo, se considera que el multilateralismo es un instrumento proveedor de legitimidad de las acciones hegemónicas de uno o varios Estados. El principal empleo de tales cuerpos multilaterales es expresar, articular y adelantar la ideología dominante hasta que adquiera hegemonía global (Taylor, 2009: 46).

Para el enfoque Constructivista, dicha caracterización pesimista del multilateralismo lo reduce a un ejercicio benevolente por parte de los Estados hegemónicos; sin embargo, es necesario situarlo en su precisa dimensión. Al respecto, Taylor enfatiza: "tal concepto estático de las organizaciones multilaterales puede ser reductivo e incluso dogmático, al confinar la trayectoria de las instituciones internacionales a una tal de simples agentes de un proyecto hegemónico dado [...]" (Taylor, 2009: 46). En términos del debate agente-estructura (Giddens, 1979; Wendt, 1987) representa supeditar al agente a la dinámica de la estructura. Igualmente, no corresponde a la evidencia empírica. En su momento, y como se mencionó, los países del Sur conformaron un bloque antiimperialista como mecanismo para la inclusión de temas afines y de trascendencia particular a dicho grupo en la agenda internacional; por ejemplo, el desarme, la descolonización o el Nuevo Orden Económico Internacional (NOEI). Al capturar temporalmente a Naciones Unidas durante los años setenta, facciones de elites del Sur fueron capaces de desarrollar la inercia necesaria para la creación de la Conferencia de las Naciones Unidas para el Comercio y el Desarrollo, UNCTAD (Taylor, 2009:46).

Desde el enfoque del Liberalismo institucional, el sistema internacional se caracteriza por la existencia de varias estructuras de poder, "diferentes según las áreas temáticas y de acuerdo a los recursos que en cada una de ellas sea posible movilizar para afectar los resultados" (Keohane, 1986: 194). Por tanto, el multilateralismo es un proceso efectivo de gobernanza global al constituirse no sólo en un mecanismo proveedor de información necesaria para los Estados en la definición de sus intereses y conducta entre sí, sino también uno de negociación para la resolución de problemas de dimensiones trasnacionales. ${ }^{7}$ Ciertamente, el enfoque Neorrealista y el Liberalismo institucional coinciden en que los Estados tienden a cooperar en función de los posibles beneficios a generarse, así como de la distribución de los costos a involucrar. Bajo ninguna circunstancia se plantea un escenario de concordia permanente; existe espacio para las discrepancias, y las ganancias producidas pueden no satisfacer las expectativas de los agentes: "es deseable que se consigan resultados tangibles, pero la ausencia de ganancias no nulifica todos los beneficios que la práctica del multilateralismo inherentemente genera" (Pouliot, 2011: 20).

En ese sentido, debe notarse que el Liberalismo institucional y el Constructivismo advierten en la estructura del sistema la existencia de fuerzas materiales, así como de ideas; producto estas últimas de la interacción entre los Estados. Las ideas se expresan en regímenes internacionales, es decir, en normas a partir de las cuales los Estados determinan su comportamiento en el sistema. "Los regímenes internacionales", define Stephen Krasner, "son los conjuntos de principios explícitos e implícitos, normas, reglas y procesos de toma de decisión en torno a los cuales concurren las expectativas de los acto-

7 El aprovisionamiento de información reduce, al mismo tiempo, la incertidumbre y la anarquía en el sistema. 
res en un área dada de las Relaciones Internacionales" (Krasner, 1982: 2). Dichas normas, establecidas por un conjunto de Estados, identifican pautas de comportamiento positivo y negativo, obligaciones y derechos, así como sanciones para quienes trasgredan las reglas.

Finalmente, el multilateralismo se practica de manera formal e informal. Se denomina formal por desarrollarse en el seno de organizaciones internacionales de carácter intergubernamental como en Naciones Unidas, la Organización del Tratado del Atlántico Norte (OTAN) o la Organización Mundial del Comercio (OMC); los compromisos adquiridos en ese marco obligan a las partes a su cumplimiento. En cambio, se denomina informal a aquel ejercido de buena fe por un grupo significativamente menor de Estados y cuyos acuerdos son asumidos sin la mediación de obligación jurídica alguna; por ejemplo, el Grupo de los Cuatro $(G-4)^{8}$, o los foros de diálogo y coordinación de IBSA, o los BRICS, como se abordará más adelante.

El multilateralismo y las normas son procesos inherentes a la gobernanza global, entendida esta última como "los procesos e instituciones, formales e informales, que guían y restringen las actividades colectivas de un grupo" (Keohane y Nye, 2000: 12). La gobernanza global es producto de la interacción no solamente del Estado y los organismos internacionales, sino también de las Organizaciones No Gubernamentales (ONG), los conglomerados trasnacionales, además de otros actores no estatales. Esa nueva interacción tiene lugar en un contexto de contestación, transformación y complementariedad de las oberanía del Estado: singularmente de su exclusividad territorial. ${ }^{9}$ Dichos procesos han configurado una cadena de coordinación minimalista a tres niveles: local o subnacional/nacional/supranacional o global ${ }^{10}$. La sesión desoberanía posibilita el funcionamiento dedicha cadena: tanto la ejecución como la aplicación de normas y acuerdos entre los Estados y los agentes no estatales en el nivel global de la gobernanza.

La globalización ha llevado, paulatinamente, a un proceso de desterritorialización y desnacionalización de la soberanía. Ambos procesos son más evidentes en la economía, como observa Sassen: "[...] los procesos globales se materializan en los territorios nacionales. Esto lleva a una necesidad de desregulación y la formación de regímenes que faciliten la libre circulación de capital, bienes, información y servicios [...]" (Sassen, 1996: xii). Desregular la economía es una demanda, especialmente de las corporaciones privadas, y sus prácticas de gobierno desafían constantemente la soberanía de los Estados. En el momento en que una empresa decide reducir costos de producción despidiendo personal en una factoría localizada fuera de su país de origen, la soberanía del Estado anfitrión se ve comprometida; sugerir a dicha empresa a revaluar su decisión es uno de los pocos recursos disponibles. "[...] la globalización bajo estas condiciones ha requerido de una desnacionalización parcial del territorio nacional y un cambio parcial de algunos compo-

8 Integrado por Alemania, Japón, Brasil e India; cuyo objeto fue presentar una propuesta conjunta de reforma a la estructura de Naciones Unidas, particularmente de su Consejo de Seguridad.

9 Existe una amplia e interesante literatura sobre la crisis del Estado en varios campos disciplinares; se recomienda particularmente los trabajos de Sassen, 1996; Hansen y Stepputat, 2001.

10 Se emplea este término de Keohane y Nye, quienes identifican en el globalismo una cadena de coordinación y/o ejecución carente de jerarquía, y minimalista en tanto que la gobernanza en su nivel global solo será aceptada si esta no excede a la nacional, puesto que su irrupción en la autonomía [soberanía] de los Estados se justifica claramente en términos de resultados cooperativos" (Keohane y Nye, 2000:14). 
nentes de la soberanía estatal a otras instituciones, desde entidades supranacionales hasta el mercado global de capitales" (Sassen, 1996: xii).

Otro ejemplo de la contestación a la soberanía territorial de los Estados se encuentra enel Protocolo de Kyoto de 1994. Este acuerdo internacional para reducir los efectos negativos provocados por el cambio climático establecía la disminución de emisiones de gases por parte de los países signatarios. La consecución de dicho objetivo requería de los Estados no solo su compromiso, sino también legislar un marco jurídico regulatorio nacional y local. Ello demandaba, igualmente, la negociación entre el Estado, las empresas y la sociedad civil. Esos dos últimos procesos generan que ciertos Estados decidan autoexcluirse de la cadena de coordinación de la gobernanza global tornándose en una cadena de coordinación minimalista. "El interés colectivo es el interés nacional, y el presidente sudafricano, Thabo Mbeki, expresó que 'el proceso de globalización necesariamente redefine el concepto y la práctica de la soberanía nacional'" (citado en Keohane y Nye, 2000: 6). Precisamente, este debate/proceso de cesión de soberanía ha sido una constante en la Política Exterior de India, como se analiza a continuación.

\section{La No alineación y el multilateralismo}

El Estado indio articuló una Política Exterior ineludiblemente influenciada por la plataforma ideológica y discursiva del Partido del Congreso Nacional Indio (Partido del Congreso): una ideología "liberal, secularista, anti-comunal, y de no violencia" (Kundra, 1956: 56). Cada uno de esos principios representaban una aspiración reivindicativa al proceso formativo del Estado, caracterizado por la violencia entre distintos grupos religiosos, falta de consenso, obstinación e intolerancia políticas, cuyo resultado fue la Partición: la creación de India y Pakistán. Por ello, la Política Exterior buscó, igualmente, construir una auto-imagen pacífica y conciliadora de la nación india, fundamentada, por un lado, en el pensamiento de Mohandas Karamchad Gandhi ${ }^{11}$, particularmente en dos conceptos empleados en el movimiento de independencia: ahimsa (no violencia) ${ }^{12}$ y purna swaraj (autogobierno, autodeterminación o autonomía). ${ }^{13} \mathrm{Y}$ por el otro, el pensamiento liberal del Primer Ministro, Jawaharlal Nehru ${ }^{14}$, quien consideraba que la política internacional debía estar basada en la observancia del Derecho y de la moral internacionales, y el Estado indio debía situarse como ejemplo de ese comportamiento: respeto a

11Gandhi se incorporó al movimiento anticolonial a mediados de la primera década del siglo XX y, aunque posteriormente renunció a la presidencia Congreso Nacional Indio, su figura y pensamiento continuaron influyendo en el movimiento de independencia.

12La estrategia de la no violencia buscó diferenciar entre la fuerza represora del Estado colonial y el carácter "pacífico" de la sociedad india. Debe advertirse, sin embargo, que el movimiento de independencia fue una lucha, y no precisamente pacífica, como el discurso oficial trata de construir. Se sugiere revisar los siguientes trabajos: Chatterjee, 1993; Khilnani, 1997; Carballido, 2005.

13 Para profundizar en las implicaciones y alcances de este concepto y el de ahimsa en la política exterior india se sugiere revisar los trabajos de Appadorai, 1981; Prasad, 1979.

14Fue uno de los principales dirigentes del Congreso Nacional Indio, posteriormente trasformado en el Partido del Congreso. Su pensamiento e incidencia aún son evidentes en las estructuras política, económica y social del país. 
la integridad y a la soberanía territorial, no agresión, equidad y beneficio mutuos; y coexistencia pacífica (Nehru, 1958; Appadorai, 1981; Kundra, 1956).

De esta forma, el Estado indio instrumentó en su estrategia de Política Exterior la promoción y el ejercicio de la cooperación internacional en el marco de los foros internacionales con dos propósitos: el primero de ellos, situar a Naciones Unidas como espacio por excelencia para la resolución de divergencias; y segundo, fortalecer la proyección de su imagen. Esa convicción fue determinante para que India sometiera a consideración del Consejo de Seguridad de Naciones Unidas (CSNU) el primer diferendo con Pakistán por la región de Cachemira (1947). ${ }^{15}$ La cuestión fue tratada por el CSNU (1948), recomendándose la convocatoria de un plebiscito popular en la región (Consejo de Seguridad, 1948: 3).

Esta primera experiencia generó un inestimable aprendizaje para diplomáticos y políticos indios. Se evidenció que "el Consejo de Seguridad es un cuerpo estrictamente político, y sus miembros toman sus decisiones sobre la base de la perspectiva de sus intereses nacionales y no de los méritos de un caso en particular" (Gharekhan, 2007: 200). ${ }^{16}$ Por tanto, cualquier otra divergencia política o territorial prevaleciente con algún otro Estado tendría que ser resuelta rigurosamente en el ámbito bilateral, evitando su internacionalización, a fin de preservar la soberanía del Estado indio (purna swaraj). ${ }^{17}$ Así, la estrategia de Política Exterior atravesó por un proceso de rectificación. Discursivamente, continuó el apoyo absoluto a la cooperación internacional, a la primacía de Naciones Unidas como foro para la resolución de disputas y a iniciativas multilaterales: la descolonización, el desarme, la no proliferación. Diplomáticamente, en cada negociación se asumiría una posición de "estricta distribución", es decir, de beneficio total. Son estos, precisamente, son los intersticios de la No alineación: una evidente tensión entre el multilateralismo y el interés nacional del Estado indio.

Ahora bien, las tensiones, las ambigüedades y las inestabilidades inherentes a la No alineación-concebida por Jawaharlal Nehru-y sus implicaciones en la Política Exterior india, particularmente en la práctica del multilateralismo, no deben ser subestimadas. Analizarla como una "fachada" para la consecución del interés nacional (Rana, 1969; Rubinoff, 1991) resulta improductivo. Igualmente, como la formación de un tercer bloque con ideales disimiles a la dinámica ideológica bipolar. Itty Abraham sugiere que, desde un primer momento, una minoría en India encontró en la práctica de la No alineación un instrumento excepcional y distintivo para adquirir poder en la política internacio-

15 La disputa territorial por la región de Cachemira tiene sus orígenes en la fase final del movimiento de independencia y en el proceso de Partición. India y Pakistán acordaron dar libertad a los principados existentes para decidir si se unían a cualquiera de los dos Estados o que estos optaran por su independencia; en esa situación estaba Cachemira. De acuerdo a las evidencias históricas, un grupo tribal descendió de las montañas del valle; el rey asumió que era un batallón del ejército pakistaní enviado para obligarlo a incorporarse a ese país. El rey solicitó ayuda a India, la cual fue negociada a cambio de que la región se incorpora como un Estado más de la Unión India (Metcalf y Metcalf, 2003:227-243; Keay, 2000: 510-515). En ese sentido, la propuesta de Nehru de llevar el asunto a la jurisdicción del CSNU fue severamente criticada por el sector más conservador del Partido del Congreso, así como por algunos partidos de la oposición, especialmente por los nacionalistas (Praja Socialist Party y el Bharatiya Jana Sangh), quienes favorecían una salida armada al conflicto.

16 El autor es diplomático de carrera y encabezó la Misión Permanente de India ante Naciones Unidas (1986-1992).

17 Este patrón conductual se transformó, paulatinamente, en una norma; el tema será abordado más adelante. 
nal, pero inapropiado e impráctico en términos reales, pues el poder se adquiere y se demuestra materialmente (Abraham, 2009). Entonces, ¿cuáles son sus presupuestos? La No alineación plantea a la interdependencia en tanto mecanismo para la generación de seguridad entre Estados militarmente débiles. "La interdependencia, en este contexto, no significa simplemente enfatizar la interacción entre países independientes. La reciente experiencia de la Segunda Guerra Mundial mostró cómo un conflicto regional fue imposible de contener en razón de las alianzas y las conexiones que entrelazan lugares remotos" (Abraham, 2009: 209). La conformación de bloques supone, entonces, una confrontación latente entre los distintos países integrantes; no existe la posibilidad de que uno de ellos asuma el papel de mediador. En cambio, si en ese escenario uno o varios países deciden mantenerse fuera de los bloques, se dan las condiciones para intermediar y/o reducir sensiblemente las fricciones entre sí. En ese sentido, Abraham observa que el análisis geopolítico indio no está basado en un modelo centro-periferia, si no uno newtoniano, es decir, de fricción entre objetos en movimiento. En ese modelo, un conflicto entre bloques es igualmente posible; la diferencia radica en la existencia de una zona de amortiguación. "Para prevenir que la Guerra Fría estallara en un conflicto militar global, era crucial que algunos países permanecieran fuera de la lucha, no alineados con ningún bloque, para proveer, precisamente, de una zona de contención en la cual las fricciones entre ambos bloques se disiparan" (Abraham, 2009: 209). Así, es posible entender que la No alineación en la Política Exterior se operó como una plataforma para la participación libre e independiente del Estado indio (purna swaraj) en la política internacional y, por tanto, se pueden comprender las reticencias a su participación en alianzas y bloques internacionales, pero también a los acuerdos que limiten su soberanía. Ello, igualmente, dificulta la consecución de la cadena de coordinación en la gobernanza global. ${ }^{18}$

No trascurrió poco tiempo para advertir dichas tensiones e inestabilidades. En la primera Conferencia Afro-asiática de Bandung (1955), India mostró una clara rivalidad política con China por el liderazgo de los países congregados. India intentó convertirse en el legítimo interlocutor entre los países descolonizados y los dos bloques; mientras que China buscó conformar una alianza política anti-imperialista. Rubinoff sugiere que las reticencias de India radicaban en:

Existen razones adicionales de por qué India, el primer país en recibir su independencia y el primero en practicar la No alineación, se encontrara a sí mismo en la irónica situación de ser el primero en resistirse a las conferencias masivas [sic. ] entre países adoptantes de similares políticas exteriores. Para empezar, los indios reconocieron que encuentros como el de Bandung proveyeron a rivales, como Pakistán, de un foro para criticar las posiciones de su país. Por consiguiente, en el futuro India estaría determinada a que mayor sustancia que el mero anticolonialismo sirviese de base para dichas reuniones. La No alineación, se esperaba, proporcionaría una mejor justificación [. . . ] (1991: s/p).

\footnotetext{
18 Debe advertirse que, si bien la No alineación fue articulada en el contexto del conflicto bipolar, no fue concebida para operar ex profeso en ese escenario. Por el contrario, el anterior análisis permite advertir su funcionalidad de larga data y en escenarios de unipolaridad e incluso de multipolaridad. De ahí que el documento que inspiró esta investigación abogue no solo por la vigencia de la No alineación, sino también por su revitalización (Khilnani, 2012; Abraham, 2009).
} 
Bandungre presentó no solo la primera reunión de países descolonizados, sino también la primera vez que se denunció el racismo, el imperialismo económico y cultural, y se defendió el derecho a la autodeterminación. Asimismo, fue la primera conferencia en la que políticos e intelectuales africanos y asiáticos articularon una respuesta al discurso hegemónico civilizatorio anglo-europeo. ${ }^{19} \mathrm{El}$ legado de esta primera conferencia, igualmente, sirvió de base para la posterior creación del MPNA, un foro formal e institucional de diálogo de aquellos países opuestos a integrarse a alguno de los bloques políticos encabezado por Estados Unidos o la Unión Soviética.

Otro ejemplo tuvo lugar durante la década de los años sesenta. India mantuvo su postura hacia la abstinencia nuclear, formando parte de las negociaciones para la firma del Tratado de No Proliferación Nuclear (TNP). Paradójicamente, el gobierno indio consideraba que un tratado de esa índole representaba una seria limitante a su soberanía, y pretendía que el Estado conservara el derecho a decir desarrollar un programa nuclear, así como su potestad de ensayar una bomba nuclear si el contexto internacional así lo requiriese. En su momento, el propio Nehru expresó sus dudas y la ambigüedad generada en los usos de la energía nuclear: "Actualmente, tendríamos que seguir a otros países en la creación de un instituto de investigación de energía atómica, no para hacer bombas, espero [...]" (Nehru, 1987: 379, cursivas agregadas).

La estrategia de estricta distribución empleada por el gobierno indio en las negociaciones consistió en exigir derechos y obligaciones equitativos tanto a los Estados poseedores de tecnología nuclear, como aquellos carentes de ella; además de salvaguardar el derecho de los Estados no nucleares a desarrollar un arsenal atómico. La intención del gobierno era evidente: exigir lo imposible, a fin de que la otra parte cediese el máximo negociable, para ser así el más beneficiado. ${ }^{20}$ EI TNP finalmente fue aprobado (1968), con el rechazo de India, quien lo calificó de discriminatorio. Al respecto, Amrita Narlikar concluye en su análisis que India desplegó la misma estrategia en las primeras negociaciones del Acuerdo General de Tarifas y Comercio (GATT, por sus siglas en inglés):

A pesar de que India es miembro fundador del GATT, sus negociadores condujeron al mundo en desarrollo a desafiar los principios centrales del estatus de la Nación Más Favorecida y de Reciprocidad, ambos pilares del acuerdo. Al proponer este desafío, India apeló a un inconfundible concepto de justicia, basado en la equidad de los resultados, en vez de la legitimidad del proceso [...] esto se tradujo en su rechazo a involucrarse en el proceso de reducción de tarifas, y en su lugar demandó ser exento de algunas obligaciones del acuerdo (mediante el Trato Especial y Diferenciando), así como del acceso a mercados de países desarrollados (a través del Sistema Generalizado de Preferencias) (Narlikar, 2006: 63).

La equidad y la justicia son dos principios de incuestionable trascendencia en cualquier acuerdo de cooperación internacional; sin embargo, en aras de favorecer la gober-

19 Para ampliar el análisis de la Conferencia de Bandung se recomienda, Appadorai, 1955; Kahin, 1956; Mackie, 2005; Pauker, 1965; Abraham, 2009.

20 Una estrategia de ese tipo puede llegar a representar un período significativo de tiempo, al grado de desgastar a las partes. En ese escenario, la parte beneficiada continúa siendo aquella a ser regulada, en este caso el Estado indio. 
nanza global, los Estados ceden soberanía, como en el caso del TNP ${ }^{21}$ y del GATT. El rechazo de India al régimen internacional de la no proliferación nuclear, muestra la ambigüedad y las inestabilidades del discurso de abstinencia nuclear, a la vez que cuestiona su compromiso con el multilateralismo y, por ende, con la gobernanza global. Mientras que, en materia comercial, representa una clara provocación al orden económico imperante. Finalmente, en esos dos eventos el Estado indio se legitimó a sí mismo frente a sus ciudadanos: la soberanía del país quedó intacta, se desafió el status quo, se reafirmó su vocación tercermundista, y se dio voz a los países con nula capacidad de negociación.

Hasta esta parte se ha tratado de notar la articulación de la No alineación como norma en el ejercicio de la Política Exterior, es decir, un marco referencial de actuación. Las normas establecen pautas regulativas de comportamiento y los agentes colaboran en su difusión. En este caso, esos agentes en la Política Exterior son aquellos políticos involucrados en su diseño, los funcionarios y diplomáticos encargados de su aplicación. ${ }^{22}$ Ahora bien, dicho marco referencial de actuación se traslada con la práctica del multilateralismo. A continuación, se analizarán las continuidades y rupturas de dicho comportamiento en la participación de India en un organismo regional y en un foro de diálogo y coordinación financiera.

\section{1. SAARC}

A principios de los años ochenta, el Sur de Asia mostraba muy poco dinamismo económico en comparación con otras regiones; por ejemplo, el Sudeste Asiático, en donde los países miembros del organismo de cooperación regional, Asociación de Naciones del Sudeste Asiático (ASEAN por sus siglas en inglés), reportaban crecimiento económico sostenido, resultado de factores internos y externos, entre ellos, la estabilidad política intra-regional. La falta de esa última variable en el Sur de Asia representaba una aparente barrera para la creación de un organismo similar.

El papel de India en la región, por otro lado, siempre ha generado suspicacias: por su preponderancia territorial, económica y militar. Sri Lanka y Bangladesh han compartido el temor de ser absorbidos por India. La relación entre Pakistán e India ha complicado, igualmente, la dinámica en la zona. Por su parte, India había mostrado poco interés en la creación de un organismo regional, y tras la invasión soviética de Afganistán (1979), se incrementó la amenaza de que una propuesta de esa índole fuera patrocinada por Estados Unidos, llevando a su involucramiento en la zona. De hecho, no se había registrado una iniciativa para, siquiera, plantear al Sur de Asia como región política y económica. "Las primeras nociones en esa materia fueron difusas [...] no fue sino hasta 1977-80 que el concepto de Sur de Asia como región se desarrolló, cuando el presidente Ziaur Rahman

\footnotetext{
21 Se encuentra irrefutable el argumento indio en contra de este tratado: es discriminatorio, desigual jurídicamente, y legitima el monopolio del uso de la energía nuclear a cinco Estados; sin embargo, en esta parte se está analizando la tensión inherente de la no alineación al ser operativa.

22 Para ampliar el estudio de las normas en la política exterior se recomienda, Ruggie, 1998; Hopf, 2002; Eyre, 1996; Axelrod, 1986; Katzenstein, 1996; Ogden, 2010; Finnemore y Sokkink, 1998.
} 
de Bangladesh sugirió que los siete países del Sur de Asia deberían trabajar en un arreglo de cooperación para mejorar los problemas económicos (Ghosh, 2013: 5 ). ${ }^{23}$

El gobierno de Bangladesh hizo llegar a sus pares en la región una propuesta inicial para la creación de un foro de cooperación regional en el sentido más amplio. Las primeras reuniones se celebraron con funcionarios de bajo nivel de Bhután, Bangladesh, India, Maldivas, Nepal, Pakistán y Sri Lanka, con el fin de evitar una excesiva politización y facilitar las actividades del comité técnico, encargado de identificar áreas de posible cooperación. Los temas de mayor relevancia y complejidad fueron reservados para los ministros de asuntos exteriores, quienes redactarían el borrador de la propuesta final a ser negociado por los respectivos Jefes de Estado y Gobierno.

India accedió a tomar parte de las reuniones por el temor a ser aislada y que el posible organismo conspirara en su contra (Ghosh, 2013; Sharma, 2001; Nuruzzaman, 1999; Baral, 1985; Kholi, 1988). No obstante, su posición fue clara: el futuro organismo regional no sería el escenario para la resolución de diferendos bilaterales. En el primer encuentro de Foreign Secretaries, realizado en Colombo (1981), el Foreign Secretary indio, S. D. Sathe señaló:

Suscribimos totalmente el punto de vista expresado en [el documento circulado por Bangladesh] que en el futuro posible la cooperación regional en el Sur de Asia tiene que ser limitada a los campos económico, técnico, científico, social y cultural [...] En esta etapa, sería útil mencionar que llegamos a un acuerdo inequívoco en lo que llamamos "reglas básicas", las cuales deben gobernar el ejercicio de la cooperación regional [entre ellas] aquí presentaré un principio a seguir escrupulosamente: evitar discusiones de asuntos bilaterales y contenciosos, y que todas las decisiones con respecto a la cooperación regional deberán ser tomadas por unanimidad (Ministry of Foreing Affairs, Government of India, 1981:127, cursivas agregadas).

Con estas tres condiciones continuaron las reuniones. Las estructuras de gobierno de la organización, denominada South Asian Association for Regional Cooperation (SA$A R C)$, perfiló a la Cumbre de Jefes de Estado y de Gobierno como la máxima estancia de toma de decisiones, seguido por el Consejo de Ministros, el Comité Permanente y los Comités Técnicos. Las áreas de cooperación identificadas fueron la agricultura y el desarrollo rural, comercio, telecomunicaciones, educación, ciencia y tecnología, salud y cultura. A ese respecto, debe mencionarse que India estaba a favor dinamizar económicamentea la región; sin embargo, Sri Lanka y Bangladesh se opusieron a ampliar el tema por temor a que llevara a una posible apertura comercial, la cual los posicionaría en desventaja frente al primero. "El abordaje de India fue sugerir [...] una división del trabajo basada en factores locales, los cuales podrían fortalecer la base económica de la región y la infraestructura. No obstante, India no forzó el tema, dado que su política era apresurar paulatinamente el proceso de cooperación regional" (Sharma, 2001:107). Finalmente, India también se resistió a dotar al nuevo organismo de una base ideológica, así como al 
establecimiento de principios o parámetros regionales a partir de los cuales se diseñara la Política Exterior de los países miembros (Kohli, 1988).

La SAARC quedó formalmente establecida en la conferencia realizada en la capital de Bangladesh, Dhaka, en 1985. En las "provisiones generales" de la Carta de la Organización se refrendó que " 1 . Las decisiones a todos los niveles deberán ser tomadas sobre la base de la unanimidad; 2. Los asuntos bilaterales y contenciosos serán excluidos de las deliberaciones" (South Asian Association for Regional Cooperation, 1985: s/p). Asimismo, se acordó que el máximo órgano se reuniría anualmente, lo cual ocurrió de manera ininterrumpida hasta 1988. A partir de la década de los años noventa y comenzado el nuevo siglo, la Cumbre se ha celebrado intermitentemente por la polarización del ambiente político.

\section{2. BRICS}

El foro de diálogo y coordinación financiera ${ }^{24}$ de los BRIC se reunió por primera vez en la ciudad rusa de Ekaterimburgo, en junio de $2009 .{ }^{25} \mathrm{El}$ objetivo fue coordinar una posición conjunta a ser presentada en la reunión del Grupo de los Veinte (G-20) ${ }^{26}$, en Londres. El planteamiento reivindicaba una reforma integral y amplia del Fondo Monetario Internacional (FMI) en el marco de la crisis financiera global:

Nos comprometemos a promover la reforma de las instituciones financieras internacionales, a fin de reflejar los cambios en la economía mundial. Las economías emergentes y en vías de desarrollo deben tener una voz más significativa, así como una mayor representación en las instituciones financieras internacionales, y sus dirigentes deben ser designados mediante un proceso abierto, transparente, cuyo proceso de selección esté basado en los méritos. (Ministry of External Affairs, Government of India, 2012).

La coyuntura de la crisis financiera de 2009 tuvo varias implicaciones políticas y económicas. Por segunda ocasión en la historia, la recesión económica tuvo su epicentro entre los países más desarrollados, generando así la desestabilización del sistema financiero y monetario global. Sólo las economías emergentes, especialmente las de China, India, y en menor grado las de Brasil, Rusia y Sudáfrica fueron capaces de mantener su crecimiento ininterrumpido, así como su liquidez. En consecuencia, los tres últimos países estuvieron dispuestos a incrementar su participación monetaria en el $\mathrm{FMI}^{27}$, a cambio

24 Los BRICS han transitado de una agenda enfocada al sistema financiero internacional a otra más amplia: desarrollo social, comercio, entre otros. Sin embargo, solamente se han materializado acciones en la esfera financiera como la creación del Nuevo Banco de Desarrollo. Razón por la cual en esta investigación se mantendrá la definición de foro de diálogo y coordinación financiera.

25 Sudáfrica fue invitado a unirse al foro hasta la reunión de Sanya, China en 2011.

26 De hecho, el G-20 es otro ejemplo de mecanismo multilateral informal, creado durante la crisis financiera de 2009 para negociar, de forma expedita y ajena a los protocolos de Naciones Unidas, una nueva ingeniería financiera capaz de mantener los flujos de capital.

27 Se realizó a través de la reasignación de los derechos especiales de giro (DEG), por un monto total de 250 mil millones de dólares, más una asignación extraordinaria equivalente a 33 mil millones de dólares (Banco de México, 2009). Cabe mencionar que el Senado de Estado Unidos aún no ha ratificado las reformas a los DEG. 
de la restructuración del proceso de elección de los miembros de la Junta de Gobierno y una redistribución del poder de voto. China se convertiría en el tercer más grande accionista del FMI, superando a Alemania, como parte de una transferencia del seis por ciento del poder de voto a las economías más dinámicas y sub representadas (Hampson y Heinbecker, 2011: 304). ${ }^{28}$

Resulta por demás señalar que los BRICS no han expresado su intención por cambiar el actual modelo de economía de mercado; en realidad, uno de los temas más importantes en su agenda es, precisamente, la reducción de las barreras al comercio. "Al respecto, nos comprometemos e instamos al resto de los Estados a resistir todas las formas de proteccionismo comercial y a luchar contra las restricciones disfrazadas al comercio. Coincidimos en la necesidad de que los resultados de las conversaciones del comercio multilateral de la Ronda de Doha sean amplios y equitativos, de tal suerte, que cumplan con su mandato de 'ronda del desarrollo'" (Ministry of External Affairs, Government of India, 2010, s/p).

Los líderes de los países integrantes de los BRICS se han reunido en ocho ocasiones $^{29}$. Los ministros de relaciones exteriores, finanzas, agricultura, salud y comercio, así como los gobernadores de los bancos centrales y los encargados de la seguridad nacional se han reunido de manera ininterrumpida desde 2008.

Asimismo, se ha creado el Foro de Negocios, la Red de Centros de Estudios, el Grupo de Investigación Económica, y el Joint Economy Study, este último comisionado a elaborar el "Informe BRICS", en el que se detallaron las áreas de sinergia y complementariedad (Ministry of External Affairs, Government of India, 2012). En la cumbre de Nueva Delhi (2012), se acordó el comienzo de las consultas para la creación de un Banco de Desarrollo encargado de "movilizar recursos para infraestructura y proyectos sustentables entre los BRICS y otras economías emergentes y países subdesarrollados, con el fin de complementar los existentes esfuerzos multilaterales e instituciones financieras regionales para el crecimiento y el desarrollo globales" (Ministry of External Affairs, Government of India, 2012).

Tras un año de negociaciones y con las dudas de India, el Nuevo Banco de DesarroIlo (NBD) fue formalmente anunciado en julio de 2014, con cien mil millones de dólares como capital base (Ministry of External Relations, Government of Brazil, 2014: s/p). Se acordó que China aportaría \$41 mil millones; India, Brasil y Rusia \$18 mil millones cada uno, y Sudáfrica $\$ 5$ mil millones (The Hindu, 2015: s/p). Su sede se ubica en Shanghai, China, y su actual presidente es el indio, K. V. Kamath.

\section{Multilateralismo minimalista}

Existe una amplia literatura para explorar teóricamente a la SAARC (Dadhich, 2005; Mukherjee-Reed, 1997; Gonslaves y Jetly, 1999, Nuruzzaman, 1999, Saéz, 2011; Haider, 2001; Murthy, 1988; Pandian, 2002; Patnaik, 2004). Ciertamente, su genealogía y desarrollo retan las premisas elaboradas por la teoría de la integración económica, además de

28 Actualmente, China es el tercer socio de FMI. Se agradece la observación a los evaluadores del artículo.

29 Ekaterimburgo, Rusia, 2009; Brasilia, Brasil, 2010; Sanya, China, 2011 (integración de Sudáfrica); Nueva Delhi, India, 2012; Durban, Sudáfrica, 2013; Fortaleza, Brasil, 2014; Ufa, Rusia, 2015 y Goa, India, 2016. 
circunscribirse en un regionalismo singular. Sería un ejercicio poco productivo observar esta experiencia en tanto poco o nada exitosa. Este organismo regional, igualmente, se ha enfrentado de manera reiterada no sólo a expectativas y reticencias políticas de sus países miembros, sino también al pesimismo académico de especialistas locales y de otras latitudes (Ghosh, 2013; Obino, 2009; Chaundhury, 2006; Chitty, 1994; Surendra, 2015; Upadhyay, 2000; Hussain, 1999; Kohli, 1988; Ahmar, 2011; Sáez, 2011). ${ }^{30}$

Más allá de dichos presupuestos analíticos, el interés metodológico ha sido ilustrar cómo la participación del Estado indio en este organismo regional puede estar supeditado al marco referencial normativo de su Política Exterior. Es decir, las ambigüedades no solo consiguen situarse en el ámbito de una estrategia para evitar la imposición de decisiones contrarias o desfavorables a los intereses regionales indios, sino también a la existencia de normas conductuales a partir delas cuales la práctica del multilateralismo se reduce a su mínima expresión.

En ese sentido, la interdependencia y el multilateralismo inherente a la SAARC ha representado, por un lado, un desafío permanente para el ejercicio de la soberanía de India: limita su libre participación al ser una estructura institucional basada en el costobeneficio colectivo cuando se trata de macrotemas: seguridad, diferendos territoriales, comercio. Por otro, evidencia-paradójicamente-cierta relevancia instrumental para India, singularmente en microtemas o áreas específicas de cooperación de menor rango, cuya ejecución y cumplimiento no comprometen a la soberanía territorial: exención de visas diplomáticas, programas para el desarrollo agrícola, acciones conjuntas para el combate a enfermedades, intercambios académicos, entre otros. Finalmente, entre un importante grupo de diplomáticos y de hacedores de Política Exterior indios aún prevalece esa visión tradicional en torno a que la permanencia como miembro de pleno derecho a un bloque tanto político como comercial, es desfavorable a los intereses indios.

La conducta de India en los BRICS no es diametralmente distinta. Las asimetrías de poder existentes en ese foro de diálogo y coordinación financiera no le son del todo favorables, teniendo en cuenta la preponderancia política y económica de China. $Y$, sin embargo, los contrapesos con el resto de los integrantes le proveen de cierto grado de maniobra; coaligándose cuando sus intereses así lo requieren. Ello se asemeja a su partición en el MNPA y en el G-77. El carácter no vinculante y de coordinación de ciertos temas financieros del foro le permite disentiry oponerse, es decir, representar el papel descrito en el esquema newtoniano, así como en la concepción de la No alineación: ser el actor libre para reducir las fricciones entre los miembros de alianzas o bloques.

En ese escenario, resulta de gran relevancia preguntarse ¿cuáles son los fines perseguidos por India en este mecanismo de coordinación? Existe una incipiente discusión al respecto, discusión de la cual han tomado parte un reducido número de especialistas del área $^{31}$. Precisamente, dichos perfiles configuran los términos de referencia de dicha

30 El papel de la SAARC resulta, igualmente, ambivalente en términos diplomáticos. A pesar de ser menospreciada por el miembro más importante, sigue siendo relevante en tanto mecanismo de presión y protesta; prueba de ello han sido las ocasiones en que Sri Lanka, Pakistán, Bangladesh y la propia India han cancelado su partición en las Cumbres de Jefes de Estado y de Gobierno en razón del contexto político regional (Obino, 2009; Patnaik, 2010).

31 Se revisaron algunos trabajos colectivos dedicados al análisis de la política exterior india y/o el nuevo papel de India en la política global para tratar de referenciar una posible discusión del tema (Sáez y Singh, 
discusión, centrada en el origen (financiero y no político), en la falta de convergencias y desencuentros entre los miembros de los BRICS. Por ejemplo, Ashok Malik nota que "[p]arte del problema con los BRICS es que parece un constructo artificial, no un bloque forjado por la guerra, convergencias políticas o sinergias económicas. Su epicentro es el triunvirato de los años noventa Rusia-India-China (RIC) propuesto por los rusos en un intento por balancear la hegemonía estadounidense [...]" (2012: s/p). Por su parte, Rajiv Bhatia observa en la "Declaración de Sanya" (2011) una suerte de posicionamiento conjunto en torno a la reforma de las instituciones financieras internacionales (Banco Mundial y Fondo Monetario Internacional), así como de Naciones Unidas. No obstante, en el contexto de la crisis de Libia se evidenciaron las diferencias: "Previo a la Cumbre de Sanya", menciona Bhatia, "los cuatro países se abstuvieron de votar la resolución en $\mathrm{N}$ [acciones] U[nidas], proveyendo así de cobertura a la intervención occidental, y uno (Sudáfrica) apoyó la resolución. En la Cumbre; sin embargo, los cinco miembros expresaron su rechazo al uso de la fuerza y el respeto al principio de no interferencia en los asuntos internos de una nación" (2011: s/p). Ese episodio demuestra no solo cuan complicado resultaría articular una postura internacional común-si acaso se ha planteado explícitamente tal cosa-, sino también las discrepancias en torno a la prevalencia y la vigencia de los principios tradicionales del derecho internacional en el marco de acciones militares conjuntas.

\section{Conclusiones}

En los dos casos abordados, con sus respectivas particularidades históricas y temporales, ha sido posible identificar los términos del debate en torno al papel de India en el contexto regional y global, e indirectamente a la propia gobernanza global. Esos términos refieren a la concepción y diseño de la Política Exterior, articulada en una estrategia poco conciliadora entre los intereses nacionales y la práctica del multilateralismo. La participación de India en la SAARCy en los BRICS, este último un foro con poco grado de institucionalización y solo compromiso retórico, ha estado supeditada normativamente a la No alineación, es decir, se da continuidad a su papel de mediador y dificulta la consecución de la cadena de coordinación en la gobernanza global.

En este artículo se ha tratado de evidenciar teóricamente, en la praxis y desde una perspectiva histórica, cómo fue articulada la política de la No alineación y cómo dicha concepción ha llevado a la práctica de un multilateralismo sumamente singular. A su vez, esa práctica dificulta, por tanto, la gobernanza global. La participación del Estado indio en un acuerdo, en un organismo regional o en un foro de diálogo y cooperación no representa en sí mismo contribuir a la gobernanza global.

En la medida en que los Estados participantes de mecanismos multilaterales o regionales transformen los compromisos adquiridos y los incorporen a los niveles nacional y local, entonces, se puede presenciar el funcionamiento de la mencionada cadena de coordinación de la gobernanza global. En el caso del Estado indio, dicha cadena de coordinación ha sido minimalista, teniendo en cuenta que es partícipe de acuerdos y regíme- 
nes globales en la medida que estos, al ser transformados y aplicados a los niveles nacional y local, no implican supeditar el ejercicio de su soberanía territorial. Así, la participación de India en la SAARC y los BRICS evidencia sus reticencias a las reconfiguraciones de la gobernanza global.

Los diplomáticos, estrategas y políticos indios coinciden en que India debe desempeñar un papel más determinante en la política global; sin embargo, no han sido capaces de articular e instrumentar una nueva estrategia para su consecución. No es por tanto paradójico que se sugiera revalorar explícitamente la No alineación. En ese sentido, debe hacerse notar que dichos actores pocas veces se refieren a India como potencia emergente. Manjari Chatterjee-Miller, sugiere que ha sido en "Occidente" donde se ha generado esa imagen/percepción (2013), la cual responde a cierto grado de ansiedad política generada desde la conclusión de la Guerra Fría y ante la falta de un contrapeso a China. Igualmente, dicha imagen/percepción ha generado una serie de expectativas sobre la Política Exterior india y los compromisos multilaterales. Por ejemplo, se ha intentado emplear intermitentemente la Carta Democrática, pero ha generado más dudas que aciertos. India, como otros países, no ha estado dispuesta a sacrificar su relación económica y comercial con aquellos países considerados poco o nada democráticos: Irán, Birmania, Arabia Saudita, Emiratos Árabes Unidos, Kazajstán.

Shashi Tharoor, diplomático indio, ha sugerido que una Política Exterior sustentada en la ética y la democracia puede representar un riesgo a la seguridad territorial y económica del país (Tharoor, 2007). Finalmente, el incremento de la participación de India en la agenda global representa mayor compromiso con la gobernanza global; cabe preguntarse si está dispuesta a ceder cada vez más soberanía. Las experiencias de India en mecanismos multilaterales como la SAARC y los BRICS han mostrado un marcado patrón normativo (No alineación y estricta distribución). A partir de ese patrón, el Estado indio persiste en caracterizar al sistema internacional newtonianamente.

\section{Referencias bibliográficas}

Abraham, Itty (2008), "From Bandung to NAM: Non-alignment and Indian Foreign Policy, 1947-65", Commonwealth \& Comparative Politics, vol. 46, núm. 2, pp. 195-219.

Abraham, Itty (2009), "Contra-proliferation: interpreting the meanings of India's nuclear tests in 1974 and 1998", en Sagan, Scott (ed.) Inside nuclear South Asia, Stanford: Stanford University Press, pp. 106-136.

Ahmar, Moonis (2011), "China in SAARC? To What Effect?: A Pakistani Perspective", Strategic Analysis, vol. 35, núm. 3, 508-510.

Appadorai, Angadipuram (1981), The domestic roots of India's foreign policy, 1947-1972Delhi: Oxford University Press.

Bajpai, K. Shankar (2013), “Knowing what's good for us” (en línea) [Fecha de consulta 14. 01. 2013]

http://www. indianexpress. com/news/knowing-what-s-good-for-us/728788

Banco de México (2009), “Asignación de Derechos Especiales de Giro (DEG) a México”, (en línea) [Fecha de consulta 24. 07. 2012]

http://www. banxico. org. mx/informacion-para-la- 
prensa/comunicados/miscelaneos/boletines/\%7BC780C160-D490-D834-96099E88BA935214\%7D. pdf

Baral, Lok Raj (1985), "SARC, but no 'shark': South Asian Regional Cooperation in perspective”. Pacific Affairs, vol. 58, núm. 3, pp. 411-426.

Baruah, Amit (2013), "Engagement yes, cravenness no". The Hindu (en línea) [Fecha de consulta 24. 01. 2013] http://www. hindu. com/2004/05/30/stories/2004053000560900. htm

Basu, Prasenjit K. et. al. (2005), India as a New Global Leader London: The Foreign Policy Centre.

Chakravorti, Bhaskar (2014), “A new club for India”. The Indian Express, August 4, (en línea) [Fecha de consulta 2. 07. 2015] http://indianexpress. com/article/opinion/columns/a-new-club-for-india/ Chatterjee-Miller, Manjari (2013), "India's Feeble Foreign Policy”, Foreign Affairs, núm. 14, pp. 14-19.

Chaundhury, Anasua Basu Ray (2006), SAARC at Crossroads, The Fate of Regional Cooperation in South Asia, New Delhi, Samskriti.

Chiriyankandath, James (2004), "Realigning India: Indian foreign policy after the Cold War", The Round Table, vol. 93, núm. 374, pp. 199-211.

Chitty, Naren (1994), Framing South Asian Transformation, New Delhi, South Asian Publishers.

Dadhich, Ramesh (2005), “Understanding SAARC: Regional cooperation in neo-colonial context". Indian Journal of Asian Affairs, vol. 18, núm. 1, pp. 49-64.

Ganguly, Sumit (ed.) (2010), India's foreign policy: retrospect and prospect New Delhi: Oxford University Press.

Ganguly, Sumit, (1991), "Review”, The Journal of Asian Studies, vol. 50, núm. 3, pp. 719-720.

Gharekhan, Chinmaya R. (2007), "India and the United Nations", en: Sinha, Atish \& Madhup Mohta (ed.), Indian Foreign Policy: Challenges and Opportunities New Delhi: Foreign Service Institute.

Ghosh, Partha S. (2013), "An enigma that is South Asia: India versus the Region". Asia-Pacific Review, vol. 20, núm. 1, pp. 100-120.

Gonslaves, Eric; Jetly, Nancy (eds.) (1999), The Dynamics of South Asia: Regional Cooperation and SAARC, London, SAGE.

González Castañeda, Mario; Bavoleo, Bárbara (2010). “Atomizando a la nación: la energía nuclear en el discurso de la nación de India". Memoria y Sociedad, vol. 14, núm. 29, pp. 125-142.

González Castañeda, Mario (2011), "La Política Exterior india: entre la continuidad normativa y la irrupción institucionalizada”, en: Oropeza García, Arturo (coord.) BRICS: el difícil camino entre el escepticismo y el asombro México, D. F.: UNAM, pp. 423437.

Hagerty, Devin T. (2005), South Asia in world politics Lanham: Rowman \& Littlefield. 
India en los márgenes de la gobernanza global: las ambigüedades en la práctica del multilateralismo (19-40)

Haider, Zaglul (2001), "Crises of Regional Cooperation in South Asia”, Security Dialogue, vol. 32, núm. 4, pp. 423-437.

Hampson, Osler; Heinbecker, Paul (2011), “The 'New' Multilateralism of the Twenty-First Century". Global Governance, núm. 17, pp. 299-310.

Harris, Jerry (2005), “Emerging Third World Powers: China, India and Brazil”. Race \& Class, vol. 46, núm. 3, pp. 7-27.

Harshe, Rajen (2005), "South Asian regional cooperation", en: Harshe, Rajen y K. M. Seethi (eds.) Engaging with the World: critical reflections on India's foreign policy New Delhi: Orient Logman, pp. 319-341.

Hussain, A (2000), "The Imperative of a Political Agenda for SAARC", en Upreti, Bhuwan Chandra (ed.), SAARC: Dynamics of Regional Cooperation in South Asia, New Delhi, Kalinga Publications, vol. 1.

India-Brazil-South Africa Dialogue Forum. "Brasilia Declaration" (en línea) [Fecha de consulta 22. 07. 2012] http://ibsa. nic. in/brasil_declaration. htm

India-Brazil-South Africa Dialogue Forum. "1st IBSA Summit Declaration, Brasilia, 2006" (en línea) [Fecha de consulta 2. 07. 2013] http://www. ibsa-trilateral. org/images/stories/documents/declarations/1st_summit_declaration. pdf

India-Brazil-South Africa Dialogue Forum. " $2^{\text {nd }}$ IBSA Summit Declaration, Tshwane, 2007" (en línea) [Fecha de consulta 22. 07. 2012] http://www. ibsa-trilateral. org/images/stories/documents/declarations/2nd_IBSA_Summit_Declaration__Tshwane_2007.pdf

India-Brazil-South Africa Dialogue Forum. "About IBSA background" (en línea) [Fecha de consulta 2. 07. 2013] http://www. ibsa-trilateral. org/about-ibsa/background

Jetly, Rajshree (2003), "Conflict management strategies in ASEAN: perspectives for SAARC". The Pacific Review, vol. 16, núm. 1, pp. 53-76.

Khilnani, Sunil. The idea of India London: Hamish Hamilton, 1997.

Khilnani, Sunil, et al (2012), Nonaligment 2. O. A foreign and strategy policy for India in the Twenty First Century New Delhi, National Defence College \& Centre for Policy Research.

Keay, John (2000). India: A History, New York: Grove Press.

Keohane, Robert (1986), "Theory of World Politics: Structural Realism and Beyond", en: Keohane, Robert (ed.), Neorealism and its critics New York: Columbia University Press.

Keohane, Robert (1989), "La teoría de la estabilidad hegemónica y los cambios en los regímenes económicos internacionales: 1967-1977", en Keohane, Robert; Instituciones internacionales y poder estatal. Ensayos sobre teoría de las Relaciones Internacionales Buenos Aires: GEL, pp. 39-54. 
Kohli, Manoram (1988), "India and South Asian regional cooperation". The Indian Journal of Political Science, vol. 49, núm. 3, pp. 301-311.

Krasner, Stephen (1983), "Structural causes and regime consequences: regimes as interventing variables", en: Krasner, Stephen (ed.) International regimes Ithaca: Cornell University Press, pp. 1-21.

Makarychev, Andrey, Morozov, Viatcheslav (2011), "Multilateralism, Multipolarity, and Beyond: A Menu of Russia's Policy Strategies". Global Governance, núm. 17, pp. 353-373.

Malik, Ashok (2012), “Alphabet soup”. Outlook, (en línea) [Fecha de consulta 15. 01. 2013]

http://www. outlookindia. com/article. aspx?280442

Mehta, Pratap Bhanu (2009), "Still under Nehru's shadow? The absence of foreign frameworks in India". India Review, vol. 8, núm. 3, pp. 209-233.

Metcalf, Barbara y Thomas Metcalf (2003). A Concise History of India, Cambridge: Cambridge University Press.

Ministry of External Affairs, Government of India (1981), "Ministry of External Affairs Record, 1981", vol. XXVII, núm. 1.

Ministry of External Affairs, Government of India (2009), "Joint Statement of the BRIC Countries Leaders, Yekaterinburg, Russia”, (en línea) [Fecha de consulta 22. 06. 2012] http://www. bricsindia. in/firstSummit. html

Ministry of External Affairs, Government of India (2010), "Joint Statement of the BRIC Countries Leaders, Brasilia", (en línea) [Fecha de consulta 22. 06. 2012] http://www. bricsindia. in/secondSummit. html

Ministry of External Affairs, Government of India (2012). "India and BRICS", (en línea) [Fecha de consulta 22. 06. 2012] http://www. bricsindia. in/india. html

Ministry of External Affairs, Government of India (2012), "Fourth BRICS Summit-Delhi Declaration", (en línea) [Fecha de consulta 22. 06. 2012] http://www. bricsindia. in/delhi-declaration. html

Ministry of External Relations, Government of Brazil (2014), "Agreement on the New Development Bank", (en línea) [Fecha de consulta 27. 08. 2015] http://brics. itamaraty. gov. br/agreements

Mukherjee, Kunal (2014), "The South Asian Association for Regional Cooperation: Problems and prospects". Progress in Development Studies, vol. 14, núm. 4, pp. 373-381.

Mukherjee, Rohan; Malone, David (2011), “From High Ground to High Table: The Evolution of Indian Multilateralism”. Global Governance, núm. 17, pp. 311-329.

Mukherjee-Reed, Anaya (1997), "Regionalization in South Asia: Theory and Praxis", Pacific Affairs, vol. 70, núm. 2, pp. 235-251.

Narlikar, Amrita (2006), “Peculiar chauvinism or strategic calculation? Explaining the negotiat- 
ing strategy of a rising India". International Affairs, vol. 82, núm. 1, p. 59-76.

Nuruzzaman, Md (1999), "SAARC and Subregional Co-operation: Domestic politics and Foreign Policies in South Asia". Contemporary South Asia, vol. 3, núm. 3, pp. 311-322.

Nye, Joseph \& John Donahue (eds.) (2000). Governance in a globalizing world, Washington, D. C.: Brookings Institution Press,

Obino, Francesco (2009), "SAARC: The political challenge for South Asia and beyond". Economic and Political Weekly, vol. 44, núm. 9, pp. 118-125.

Pandian, S. G. (2002), “Moving South Asia's Economies beyond the Indo-Pakistan Paradigm in the South Asian Regional Association for Cooperation", Contemporary South Asia, vol. 11. , núm. 3, pp. 32-344.

Pant, Harsh V (2008), India negotiates its rise in the international system New York: Palgrave.

Pant, Harsh V (2009), "Indian foreign policy challenges: substantive uncertainties and institutional infirmities". Asian Affairs, vol. 50, núm. 1, pp. 90-101.

Pant, Harsh V. (ed.) (2009), Indian foreign policy in a unipolar world, New Delhi: Routledge.

Patnaik, Smruti S. (2004), "Indo-Pak Relations and the SAARC Summits”, Strategic Analysis, vol. 28, núm. 3, pp. 427-439.

Patnaik, Smruti S. (2010), "SAARC at 25: An Incredible Idea still in its Infancy", Strategic Analysis, vol. 34, núm. 5, pp. 671-677.

Perkovich, George (2000), “What makes the Indian bomb tick?”, en: Sar Desai, D. R. (ed.). Nuclear India in the Twenty-First century New York: Palgrave, pp.

Pouliot, Vincent (2011), "Multilateralism as an End in Itself". International Studies Perspectives, núm. 12, p. 18-26.

Prasad, Bimal (ed.) (1979), India's foreign policy: studies in continuity and changeNew Delhi: Vikas.

Raja, Mohan C. (2004), Crossing the Rubicon: the shaping of India's new foreign policy, New York: Palgrave Macmillan

Rubinoff, Arthur G (1991), "The multilateral imperative in India's foreign policy”, Round Table, vol. 31, núm. 9, s/p.

Ruggie, John (1992), "Multilateralism: The Anatomy of an Institution". International Organization, núm. 46, pp. 561-598.

Sáez, Lawrence (2011), The South Asian Association for Regional Cooperation (SAARC): An Emerging Collaboration Architecture, London, Routledge.

Sikri, Rajiv (2009), Challenge and strategy New Delhi, SAGE.

South Asian Association for Regional Cooperation. "SAARC Charter", 1985 (en línea) [Fecha de consulta 2. 07. 2015]

http://www. saarc-sec. org/SAARC-Charter/5/

Sharma, Suman (2001), "India and SAARC". India Quarterly, vol. 57, núm. 2, pp. 99-120.

Surendra Kumar, S. Y. “China's SAARC Membership: The Debate”, International Journal of 
China Studies, vol. 6, núm. 3, pp. 299-311.

Tharoor, Shashi (2007), “Can India afford an ethical foreign policy?” (en línea) [Fecha de consulta 12. 01. 2013] http://articles. timesofindia. indiatimes. com/2007-10-14/all-thatmatters/27996147_1_foreign-policy-uncomfortable-question-pandit-nehru

Taylor, Ian (2009), "The South will Rise Again? New Alliances and Global Governance: The India-Brazil-South Africa Dialogue Forum". Politikon, vol. 36, núm. 1, p. 45-58.

The Hindu (2015), "The building of the BRICS bank". The Hindu, May 18, (en línea) [Fecha de consulta 2.07. 2015] http://www. thehindu. com/business/the-building-of-the-bricsbank/article7214558. ece

Waltz, Kenneth (1979), Teoría de la Política Internacional Buenos Aires: GEL.

Waltz, Kenneth (2000), "Structural Realism after the Cold War". International Security, vol. 25, núm. 1, pp. 5-41.

Wendt, Alexander (1987), "The Agent-Structure Problem in International Relations". International Organization, vol. 41, núm. 3, pp. 335-370.

Yahya, Faizal (2004), "Pakistan, SAARC and ASEAN Relations". Contemporary Southeast Asia, vol. 26, núm. 2, p. 346-75. 